\title{
Advanced cervical cancer in young women: imaging study of late and very late radiation-related side effects after successful treatment by combined radiotherapy
}

\author{
Hana Malikova ${ }^{1,2}$, Miroslava Burghardtova ${ }^{1}$, Klara Fejfarova ${ }^{1}$, Katarina Nadova $^{1}$, Jiri Weichet ${ }^{1}$ \\ ${ }^{1}$ Radiology Department, Third Faculty of Medicine, Faculty Hospital Kralovske Vinohrady, Charles University, Prague, Czech Republic; ${ }^{2}$ Institute \\ of Anatomy, Second Medical Faculty, Charles University, Prague, Czech Republic \\ Correspondence to: Hana Malikova. Department of Radiology, Third Faculty of Medicine, Faculty Hospital Kralovske Vinohrady, Charles University, \\ Srobarova 1150/50, 11000 Prague, Czech Republic. Email: hana.malikova@fnkv.cz.
}

Background: Radical combined radiotherapy (RT) is a standard treatment for advanced cervical cancer. The aim of our study was to identify morphological late ( $\geq 6$ months) and very late ( $\geq 5$ years) radiationrelated comorbidities on computed tomography (CT), positron emission tomography/computerized tomography (PET/CT) or magnetic resonance imaging (MRI) scans in young females who survived $\geq 5$ years since RT and were considered as successfully treated. Secondly, we studied a rate of clinically silent radiationrelated toxicities apparent on imaging scans that might influenced on future well-being of survived females. Thirdly, we analyzed reasons why patients underwent imaging scans.

Methods: We included 41 subjects aged under 50 years (mean $41.8 \pm 7.2$ years, median 41 years), who survived $\geq 5$ years since RT, with at least one available imaging scan $\geq 3$ years since RT. The mean time between RT and the last available scan was $12.3 \pm 7.9$ years (median 9 years); the mean time of clinical followup was $15.4 \pm 7.1$ years (median 14 years).

Results: Fourteen (34.1\%) females underwent the first imaging scan in emergency situation and 27 (65.9\%) patients due to variable chronic complaints. Grade III-V radiation-related comorbidities occurred in 19 (46.3\%) females including one case of death due to radiation-induced osteosarcoma. In 14 of 19 patients, comorbidities were multiple. Four (9.8\%) females suffered from life-threatening complications (grade IV): one from an uretero-arterial fistula with massive hematuria and 3 from bowel perforations. Eleven (26.8\%) subjects suffered from bowel strictures with ileus development, they underwent mean $1.7 \pm 0.8$ surgery (median 1.5). The mean time since RT to the first surgery for intestinal complications was $5.4 \pm 6.0$ years (median 3 years), to the second $12.0 \pm 9.6$ years (median 11.5 years) and to the third surgery $9.0 \pm 4.2$ years (median 9 years). Late fistulas formations (vesico-recto-vaginal) were seen in $6(14.6 \%)$ patients. Bone complications were diagnosed in $9(22.0 \%)$ of treated females, one case of osteosarcoma included, 4 of 9 bone complications were clinically silent. In $5(12.2 \%)$ subjects, toxicities grade III-IV first time manifested $>5$ years since RT, majority of them were multiple. The bowel perforation and fistulas formations were the earliest manifested grade III-IV toxicities, with median time 3 years since RT, the bone complications were the latest manifested with median time 16 years since RT.

Conclusions: Our study is not able to bring information about the incidence of late and very late radiation related comorbidities, according to International Federation of Gynecology and Obstetrics (FIGO) recommendations patients are clinically followed only for 5 years and imaging cross-sectional scans are not recommended. However, our study shows that if females successfully treated for advanced cervical cancer report abdominal/pelvic clinical problems, it is highly probable their imaging scans will reveal late radiation related side-effects that may affect the rest of their life. It puts forward question whether females after radical RT should been regularly followed by cross-sectional imaging methods. 
Keywords: Radiation-induced tumor; complication; bone; toxicity; arterio-ureteral fistula

Submitted Apr 09, 2020. Accepted for publication Jun 10, 2020.

doi: 10.21037/qims-20-553

View this article at: http://dx.doi.org/10.21037/qims-20-553

\section{Introduction}

Although the incidence of cervical cancer has been declining last years in developed countries, this cancer is still the fourth most common cancer in women worldwide (1). In 2012 , it was estimated that cervical cancer was responsible for 265,700 deaths worldwide (1). The decline of the incidence in western countries is caused by cervical screening programs and also by anti-human papilloma virus vaccinations $(1,2)$. Unfortunately, cervical cancer still remains the seventh most common cancer overall and the leader in cancer-related death in women under the age of 35 years $(1,2)$.

Cervical cancer is staged according to the International Federation of Gynecology and Obstetrics (FIGO) system (2). In early stages of the disease, the most preferable treatment is surgery; however, for advanced cancer stages IIB and higher the chemoradiotherapy is the main option for therapy $(2,3)$. Radiotherapy (RT) usually includes external beam radiation and intracavitary brachytherapy (2). It is well known that combined RT brings a wide spectrum of radiation-related side-effects; those, which develop within 6 months since finishing RT are considered as acute, later than 6 months since RT are considered as late. Bone marrow, bowels and the urinary bladder are the most radiation sensitive tissues and organs in the field of RT. Proctocolitis, enteritis and cystitis are common acute comorbidities; however, radiation-related pathological processes may result in different fistulas, enteral/colic and ureteral strictures with hydronephrosis development, ileus or bowel perforation (3). Fatty-replacement of bone marrow is an inevitable result of RT; in accented cases RT may lead to osteonecrosis and insufficiency bone fractures (3). Last, but not least, radiation-induced tumors must be mentioned among very late radiation-related complications, which appear usually more than 5 years after RT (4). Acute radiation-related comorbidities are well known and expected; however, late and especially very late comorbidities later than 5 years since RT may escape from our attention; according to the FIGO report 2018 the clinical follow-up is recommended for 5 years since RT and routine imaging scans are not indicated. The patients are considered successfully cured after 5 years disease-free period and should be returned to the standard screening program (2).

The above-mentioned recommendation led us to an imaging study. Its aim was to identify morphological late and very late radiation-related side effects detectable on computed tomography (CT), positron emission tomography/computerized tomography (PET/CT) or magnetic resonance imaging (MRI) scans in young females who survived more than 5 years since RT and were considered as successfully treated for advanced cervical cancer. We aimed at young females, because late toxicities may seriously affect the rest of their life. Secondly, we intended to study a rate of clinically silent or minimally manifested radiation-related toxicities apparent on imaging scans that might influence on the future wellbeing of successfully treated females. Thirdly, we analyzed indications of very late imaging scans as it is not the standard of care according to FIGO recommendation (2).

\section{Methods}

This study was designed as retrospective. We searched the hospital information system and selected all females with the history of advanced cervical cancer treated by radical RT or chemoradiotherapy, who visited for any reason the Oncology Department of our institution during the years 2011 and 2012. From those patients, we selected the subjects, who underwent any imaging cross-sectional scans (CT, PET/CT or MRI) and reviewed all their medical records since their cancer diagnosis to the end of the year 2018. The study group was selected according to the following inclusion criteria:

(I) Age under 50 years in the time of cervical cancer treatment;

(II) At least 5 years survival since RT with complete clinical data for reviewing;

(III) At least one available imaging scan (CT, PET/ CT or MRI) $\geq 3$ years since RT. Patients who were followed-up only by vaginal and abdominal 
Table 1 Common terminology criteria for adverse events v5.0 (5)

\begin{tabular}{ll}
\hline Grade & Simplified criteria of radiation-related adverse events \\
\hline Grade I & Mild; asymptomatic or mild symptoms, clinical or diagnostic observation only; intervention is not indicated \\
Grade II & Moderate; minimal, local or non-invasive intervention indicated; limiting age propriate instrumental ADL \\
Grade III & $\begin{array}{l}\text { Severe or medically significant but not immediately life-threatening; hospitalization or prolongation of hospitalization indicated; } \\
\text { disabling; limiting self-care ADL }\end{array}$ \\
Grade IV & Life-threatening consequences; urgent intervention indicated \\
Grade V & Death related to adverse effect
\end{tabular}

ADL, activities for daily living.

ultrasound and chest $\mathrm{X}$-rays were not involved in the study.

We reviewed all available clinical and imaging data and evaluated the following imaging signs on CT, MRI, PET/ CT scans:

(I) Chronic cystitis (signs of fibrotic wall thickening or wall edema and wall irregularities on MRI or CT);

(II) Distal ureteric strictures with upstream dilatation of the ureter and hydronephrosis;

(III) Chronic enteritis and proctocolitis (bowel wall thickening, stranding, bowel wall edema, etc.) and their complications as bowel strictures, ileus development and/or bowel perforation;

(IV) All visible fistulas (entero-enteral, vesico-enteral, vesico-vaginal and others);

(V) Musculoskeletal complications as osteonecrosis and insufficiency fractures;

(VI) Radiation-induced secondary malignancy;

(VII) Mucometra resulting from vaginal or cervical synechia;

(VIII) Any other severe complications or findings that may be related to RT.

Only findings that appeared $\geq 6$ months since RT were assessed as the late radiation-related toxicities or side effects, radiation-related comorbidities presented $\geq 5$ years since RT were assessed as very late.

The clinical relevance of radiation-related comorbidities, patients' complaints and imaging findings were graded according to the common terminology criteria for adverse events v5.0, simplified criteria of grading system are summarized in the Table 1 (5).

Data were expressed as mean \pm SD and median. Analyses were performed using STATISTICA software version 12.

The study was performed in accordance with the
Declaration of Helsinki and was approved by the local ethics committee, Faculty Hospital Kralovske Vinohrady, Prague, Czech Republic. All patients provided written, informed consent and agreed with publishing medical data in scientific literature in anonymous form.

\section{Results}

\section{Patient selection}

The retrospective study was initiated in the year 2018; we identified 91 females, aged $41.8 \pm 7.2$ years (median 41 years) that visited our cancer center during the years 2011 and 2012 and were treated for advanced cervical cancer by radical RT/chemoradiotherapy in history. Thirtyseven of them did not survive 5 years since RT or were lost from the hospital database; 54 females survived more than 5 years and were considered as successfully treated. Of them, we excluded patients, who did not fulfill the rest of the inclusion criteria (mostly lack of imaging scans $\geq 3$ years since RT); 41 females fulfilled all inclusion criteria and were enrolled into the study.

All included patients underwent more than one CT, MR or PET/CT scan; the last available scans were performed $12.3 \pm 7.9$ years (median 9 years) since RT. The main reasons for the first imaging scan were following: 14 (34.1\%) females were scanned in emergency conditions (12 of them with acute abdomen symptoms, one for a unbearable hip pain, one for sepsis and suspicion for spondylodiscitis), the rest of them ( 27 females, $65.9 \%$ ) were scanned for multiple chronic complaints (one for progressive hip/back pain, 16 females underwent scans for different unspecific, mostly multiple pelvic complains as vaginal discharge/intermittent bleeding and/or chronic abdominal/pelvic pain and/or dysuria or other urinary problems mostly in combination with unspecific enteral/colic/rectal complaints). All selection 
Table 2 Patient selection and demographic data

\begin{tabular}{ll}
\hline Variables & Value \\
\hline Number of females & 41 \\
Age & Mean $41.8 \pm 7.2$ years; median 41 years \\
Time of imaging follow-up (CT, PET/CT, MRI) & Mean 12.3 \pm 7.9 years; median 9 years \\
Time of clinical follow-up & Mean $15.4 \pm 7.1$ years; median 14 years \\
Survived to $2018^{\ddagger}$ & 37 patients \\
Non-survived up $2018^{\S}$ & 4 patients (3 late cancer recurrence, 1 RT induced osteosarcoma) \\
Time between RT to pts death & Mean 15.2 \pm 7.0 years; median 16.5 years \\
Chemotherapy underwent & 27 patients \\
Histological origin of cervical cancer & 40 epidermoid carcinomas; 1 adenocarcinoma \\
\hline
\end{tabular}

${ }^{\dagger}$, time of imaging follow-up = the last available CT, PET/CT or MRI scan; ${ }^{\ddagger}$, number of females who survived since RT to the time of a study initiation; ${ }^{\S}$, number of females who did not survive to the time of a study initiation and died for different reasons (mentioned in the brackets). CT, computerized tomography; PET/CT, positron emission tomography/computerized tomography; RT, radiotherapy.

and demographic data are summarized in the Table 2.

\section{Radiation-related side effects/toxicities found on imaging scans}

In $11(26.8 \%)$ females imaging scans were negative with respect to radiation-related side effects. In $11(26.8 \%)$ patients only minimal or minor side effects (grade I-II) were found. The most interesting case of them was an extreme mucometra discovered 24 years after RT; the patient suffered from mild chronic abdominal tenderness and a minimal surgical intervention was needed for her recovery (Figure 1).

Grade III-V radiation-related toxicities occurred in $19(46.3 \%)$ females, 14 of them suffered from multiple comorbidities. Only in 5 subjects a solitary grade III-IV toxicity was found (one patient suffered from large bowel perforation, 3 females from ileus due to small bowels strictures, one from massive urinary bladder cystitis with bilateral hydronephrosis which were treated by bilateral nephrostomy). One patient died of undifferentiated bone sarcoma in the site of iliac osteonecrosis (for more details see the Case report 1). Four (9.8\%) females suffered from life-threatening complications (grade IV): one case of an uretero-arterial fistula with massive hematuria requiring an urgent vascular intervention was found (for more details, see also the Case report 2); 3 females suffered from bowel perforation and peritonitis (one of them was the patient who died of radiation induced osteosarcoma). In 11 (26.8\%) subjects, serious intestinal radiation-related complications (grade III) with ileus development were found (2 of them was operated also for bowel perforation mentioned above). Affected patients underwent minimally one surgery, and maximally 3 urgent operations (mean $1.7 \pm 0.8$; median 1.5 ). The mean time since RT to the first surgery for intestinal complications was $5.4 \pm 6.0$ years (median 3 years), to the second operation $12.0 \pm 9.6$ years (median 11.5 years) and to the third surgery $9.0 \pm 4.2$ years (median 9 years). The diagnosis of radiation-related enteritis/colitis was proved histologically in all cases. In 4 of 12 subjects the first clinical/imaging manifestation (ileus or perforation requiring surgery) developed more than 5 years since RT (mean time $12.5 \pm 5.0$ years, median 13.5 years).

Late fistulas formations were depicted in $6(14.6 \%)$ patients, they were multiple in 4 patients (one case of multiple entero-enteral fistulas, 3 cases of combined vesico-vaginal and recto-vaginal fistulas) (Figure 2). The most serious case was an uretero-arterial fistula developed 10 years since RT (see also the Case report 2), this female suffered also from combined vesico-vaginal and rectovaginal fistulas. All fistulas formations caused serious clinical problems, all required surgical or other interventional treatment.

Bone complications were found in $9(22.0 \%)$ treated females; solitary in 2 cases, multiple osteonecrosis and/ or insufficiency fractures in other cases. Four of 9 cases of bone complications were clinically silent.

Overall, in $5(12.2 \%)$ subjects severe late toxicities first time manifested $\geq 5$ years since RT, most of them were multiple (usually a combination of genitourinary and 

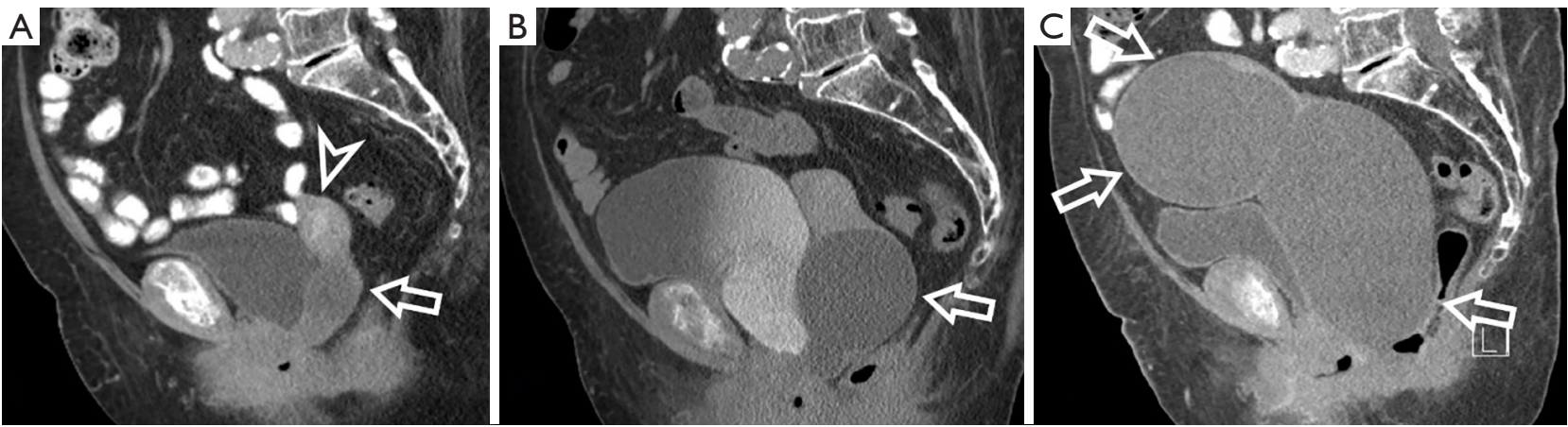

Figure 1 Mucometra formation due to radiation-related synechiae caused vaginal stenosis. Mucometra started to develop 24 years since radical RT for cervical cancer; the CT scan (A) showed the slightly dilated upper vagina filled in with hypodense mucus (arrow), the uterus looked normal (arrowhead). There was a progression of the vaginal and cervical canal dilatation (arrow) on the CT scan 16 months later (B). Another 22 months later CT showed further dilatation of the vaginal and cervical canal as well as the enlarged uterus filled with hypodense mucus (arrows, C). RT, radiotherapy; CT, computed tomography.
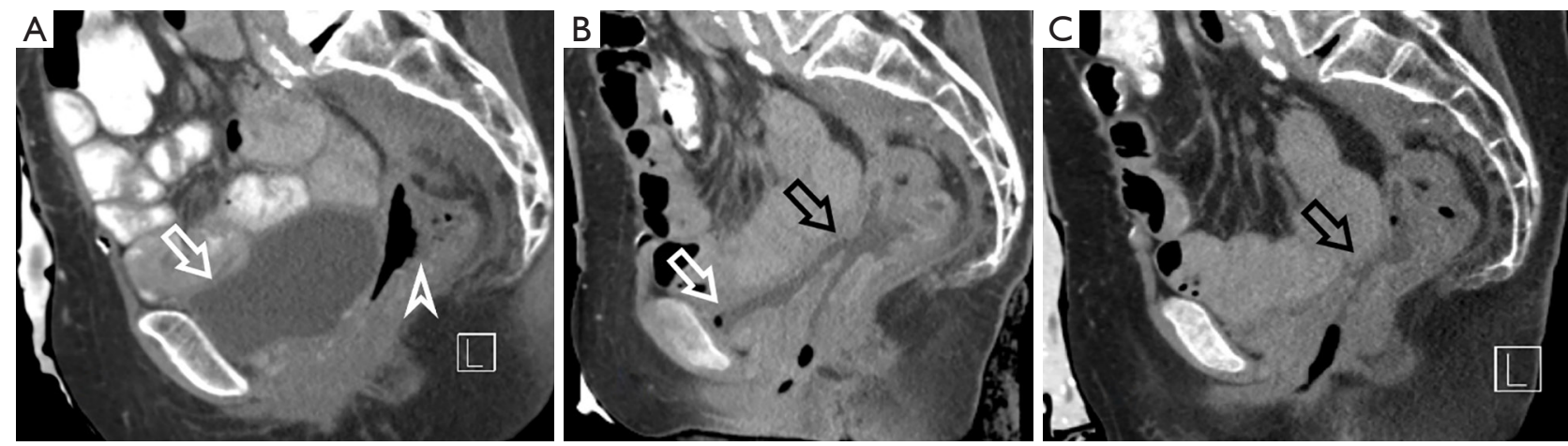

Figure 2 Complex recto-vagino-vesical fistula. (A) A rectovaginal fistula (arrowhead) was apparent on the CT scan 5 years after RT. Note normal appearance of the urinary bladder (arrow). (B) Five months later an air bubble was present in the urinary bladder (white arrow), a vesico-vaginal as well as recto-vaginal fistulas were evident (black arrows). (C) Both fistulas were still visible on CT follow-up 4 years later (arrow). RT, radiotherapy; CT, computed tomography.

gastrointestinal toxicities).

More details are presented in the Tables 3,4. The Table 3 shows that the bowel perforation and fistulas formations were the earliest manifested grade III-IV toxicities, with median time 3 years since RT, the bone complications were the latest manifested with median time 16 years since RT.

\section{Case report 1: secondary induced osteosarcoma as very late complication of radical $R T$ for advanced cervical cancer}

A 36-year-old woman with advanced cervical cancer was treated by radical combined RT and chemotherapy in 1992. The first CT scan was indicated in 2000,8 years after RT, because she was complaining of unbearable hip pain.
CT described radiation-related necrosis of the right iliac bone and insufficiency fracture of the sacrum. In 2003 she visited emergency department for acute abdominal pain and the CT scan showed pneumoperitoneum, ileus and small bowel perforation. She underwent acute surgery with ileum resection. The histology revealed radiation-related strictures. Next CT scan was indicated for unbearable pain of the right hip in 2009. It described pathological fracture of the right iliac bone, probably in the terrain of osteonecrosis. In 2011 she came again with clinical signs of acute abdomen, there was ileus of the large bowel with a sigmoid colon stricture on CT scans. Emergency surgery was performed and post-irradiation fibrosis of the sigmoid wall with severe stricture was histologically 
Table 3 Late radiation-related side effects ( $\geq 6$ months since radiotherapy)

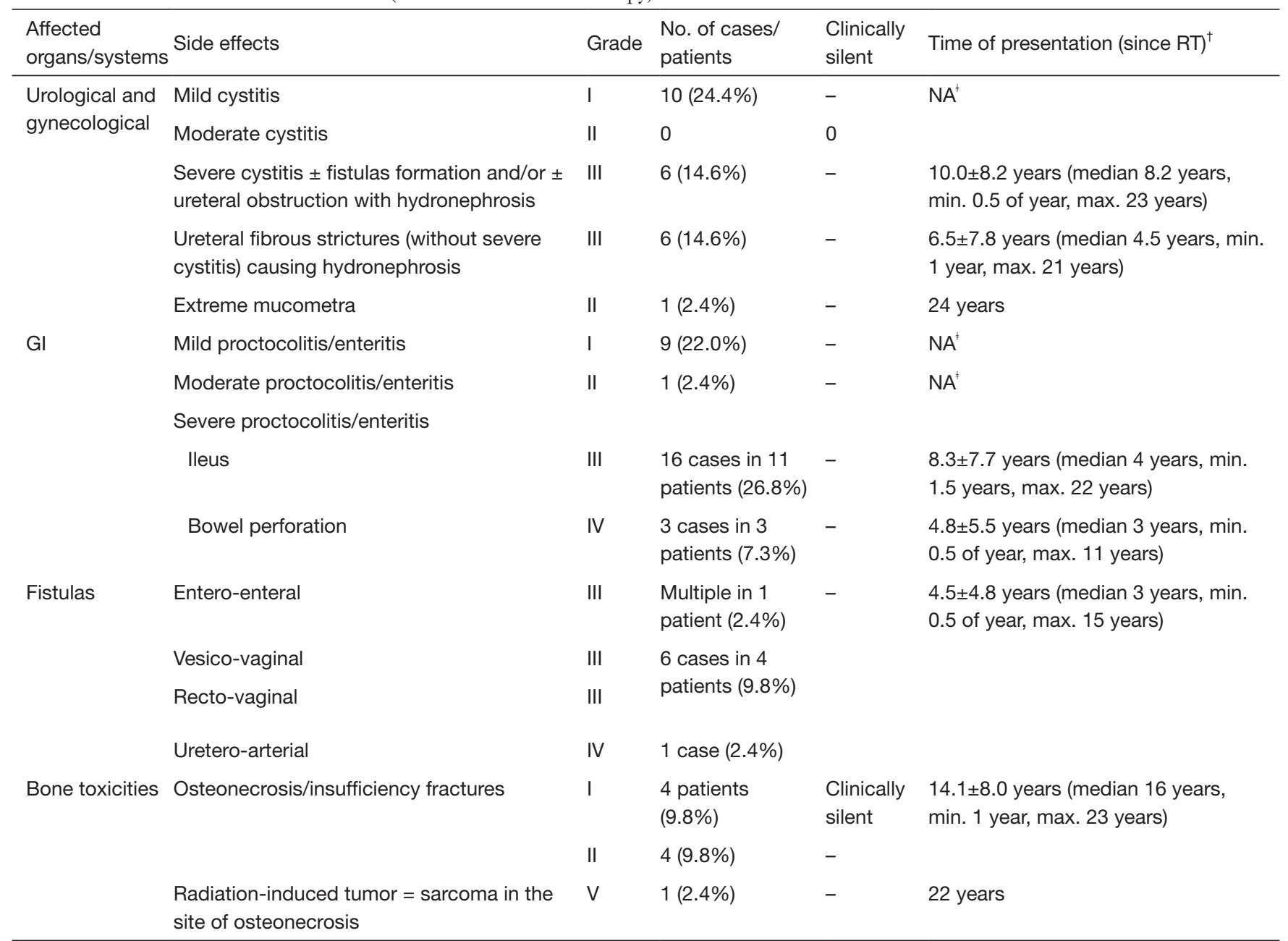

\footnotetext{
${ }^{\dagger}$, time of presentation on imaging scans; ${ }^{\ddagger}, \mathrm{NA}$, not assessed, it means that signs were apparent or clinically present during whole follow-
} up. Gl, gastrointestinal; No., number; RT, radiotherapy.

Table 4 Severe late toxicities according to time of presentation

\begin{tabular}{lcc}
\hline Severe late toxicities & $\geq 6$ months $^{\dagger}$ & Grade III-V side effects \\
\cline { 2 - 3 } $\begin{array}{l}\text { Severe cystitis, fistulas formation or ureteral } \\
\text { obstruction }\end{array}$ & $6(14.6 \%)$ patients & $4(9.8 \%)$ patients \\
Fibrous ureteral strictures & $6(14.6 \%)$ patients & $2(4.9 \%)$ patients \\
lleus/bowel perforation & 19 cases in $14(34.1 \%)$ patients & 8 cases in $6(14.6 \%)$ patients \\
$\begin{array}{lcc}\text { Different fistulas formations (multiple included) } \\
\text { Severe bone complications }\end{array}$ & $7(17.1 \%)$ patients & $2(4.9 \%)$ patients \\
Radiation-induced tumor & $1(2.4 \%)$ patient & $1(2.4 \%)$ patient \\
\hline
\end{tabular}

${ }^{\dagger}$, number of affected patients/cases found $\geq 6$ months since RT to the end of follow-up; ${ }^{\ddagger}$, number of affected patients/cases found more than 5 years since RT as the first manifestation of late toxicities. RT, radiotherapy. 

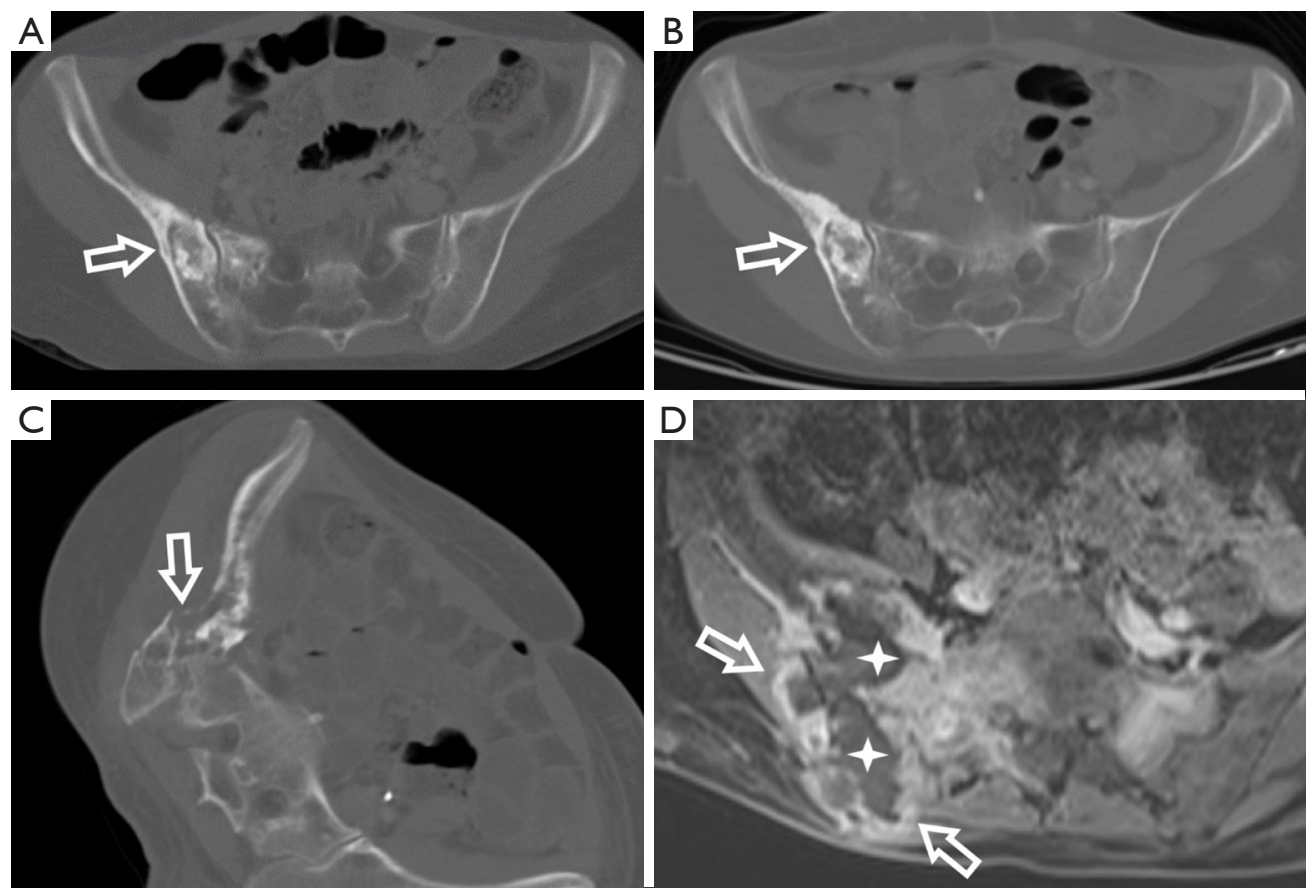

Figure 3 Radiation induced osteosarcoma. Sixteen years after combined RT for cervical cancer, right iliac osteonecrosis was present on the CT scan (arrow) (A). It was stable on CT follow-up 5 years later (B). Another year later she suffered from severe low back pain, she was able to undergo CT only in non-standard position (C). There was an evident progression of the osseous lesion with pathological fracture (arrow). MRI, a T1 weighted gadolinium enhanced fat saturated image (D), showed malignant lesion with rim enhancement (arrows) and central necrosis (asterisk).

proven. Also signs of healing fracture in the right iliac bone were present on CT in that time. Next 3 years her clinical status was stable, she was followed-up by PET/CT scans with satisfied results. In 2014 she was driven by her family to the emergency department with unbearable right hip and leg pain, complained also about back and abdominal pain; she was unable to walk and was in fever. The CT scan revealed the large progressive right iliac bone lesion, containing osteolytic and sclerotic portions with the soft tissue mass spreading out of the bone. The soft tissue mass invaded into the distal ureter and to the urinary bladder causing hydronephrosis and urosepsis. Multiple lung metastases were found. An autopsy proved undifferentiated osteosarcoma. We suggest sarcoma was induced by previous RT considering above-mentioned data. See also Figure 3 for more details.

\section{Case report 2: external iliac artery pseudoaneurysm causing life-threatening hematuria}

A 50-year-old female underwent radical RT and chemotherapy for cervical cancer in 2006. She suffered from radiation-induced colitis and cystitis with vesicovaginal and recto-vaginal fistulas in post-treatment period. Bilateral ureteral strictures developed and were treated by ureteral pig-tail stents placement on both sides in 2012 . In December 2016 she was admitted to our hospital with urosepsis and the right ureteral pig-tail malfunction. A right kidney nephrostomy had to be performed. Deep vein thrombosis in the right femoral vein was found and low molecular weighted heparin treatment was started in the same time, the patient was then discharged from the hospital to home care. Four days later after the nephrostomy procedure the patient came back to the hospital with gross hematuria via right kidney nephrostomy and serious anemia (hemoglobin $68 \mathrm{~g} / \mathrm{L}$ ). The interventional radiologist was asked to perform a digital subtraction angiography (DSA) immediately to find the source of bleeding and treat it endovascularly if possible. DSA showed pseudoaneurysm in the right external iliac artery close to the right ureter, contrast leak and filling of the ureter along the pigtail; arterio-ureteral fistula was detected (Figure 4). 

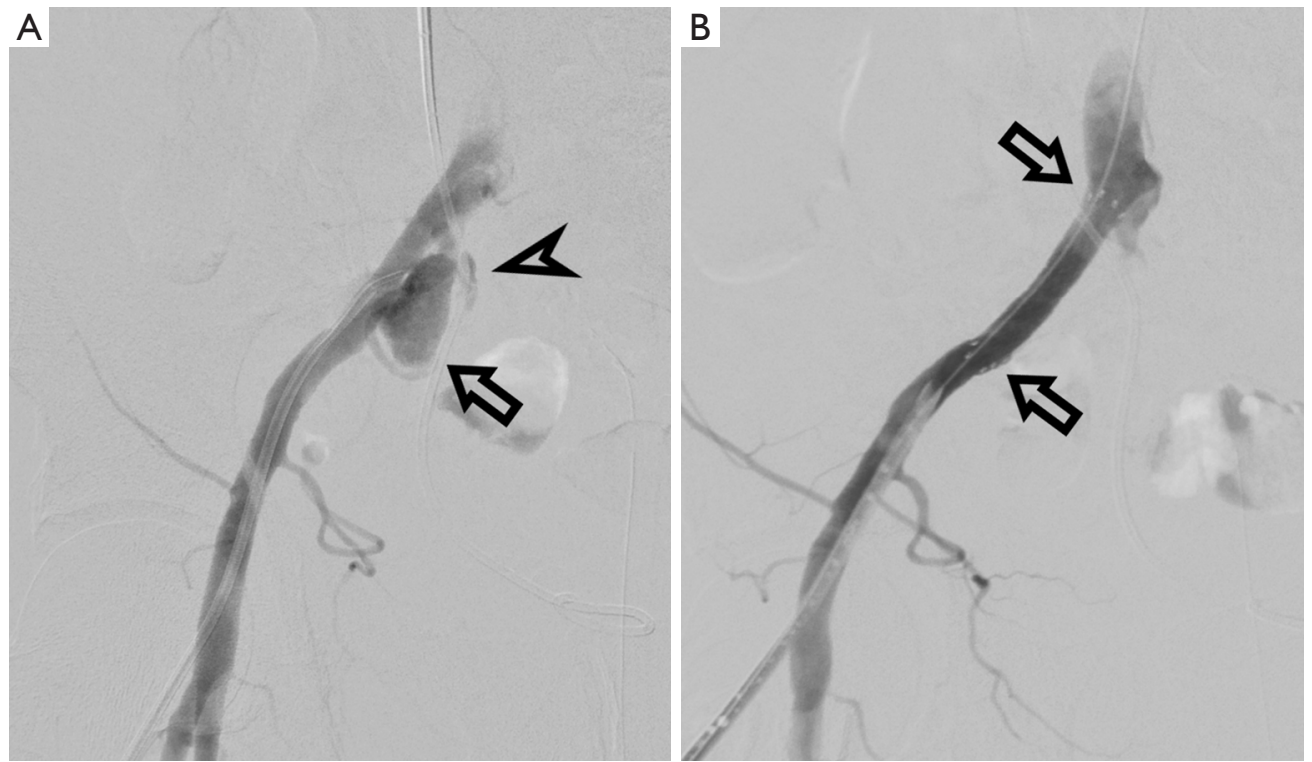

Figure 4 Arterio-ureteral fistula. Ten years after RT, the patient suffered from severe hematuria. DSA showed pseudoaneurysm of the right external iliac artery (arrow) with contrast filling of the adjacent ureter (arrowhead), DSA proved the arterio-ureteral fistula (A). The pseudoaneurysm and fistula was excluded after the covered stent placement [arrows at end marks of stent graft (B)].

Consequently, a covered stent was placed into the external iliac artery over the pseudoaneurysm origin; DSA followup confirmed the closure of the pseudoaneurysm and the arterio-ureteral fistula (Figure 4). After the procedure the hematuria ceased, the blood volume was replenished using crystalloids solutions and blood transfusions. She was discharged from the hospital 8 days since the stenting. No hematuria or anemia recurrence was detected in follow-up 1 month later.

\section{Discussion}

According to FIGO recommendation, females treated for cervical cancer are clinically followed for 5 years, then they should be returned to the standard screening program, CT or other scans (MRI, PET/CT) are not generally recommended (2). Therefore, it is complicated to collect sufficient data for the incidence study of very late (more than 5 years since RT) radiation-related comorbidities. Moreover, patients are very often lost from cancer center follow-up even in the first 5 years after therapy; some of them may die due to other diseases, some of them go well and do not want to be followed, some of them probably change an oncologist/a gynecologist for different reasons and probably partly return to the common screening program in other institutions. In our retrospective study we were not able to assess the incidence of late/very late radiation-related side effects due to the design of the study. The aim of the study was to assess very late radiationrelated toxicities in young females who were successfully treated for advanced cervical cancer, survived RT more than 5 years, reported different clinical complaints or symptoms and were sent to cross-sectional imaging scans. That means our study group was highly selected; however, we collected clinical and imaging data from a remarkable long period, the mean clinical follow-up in the study group was about 15 years (median 14 years), the imaging follow-up about 12 years (median 9 years). We aimed at young females due to their long-life expectancy after successful cancer treatment; late radiation-related toxicities in that group of patients may seriously affect their rest of life and well-being.

Most of our selected patients were sent to imaging crosssectional scans due to different chronic, usually unspecific complaints; however, 14 (34.1\%) of them underwent the first CT scan for acute or emergency conditions. In our group of patients, the late radiation-related toxicities grade III-V appeared in nearly half (46\%) of subjects and were mostly multiple. Different late toxicities were found during the whole time of the follow-up, in $12 \%$ of patients, serious late toxicities developed more than 5 years since RT. Unfortunately, our results show that late toxicities may significantly affect the rest of life of young females, who 
were successfully treated for advanced cervical cancer by radical RT.

A comparison of our results with other literary data is not easy especially due to the design of our study and very long time of clinical and imaging follow-up of our females. The most of other studies published data from relatively shorter time of follow-up, usually 3-5 years. Those studies also did not provide data from cross-sectional imaging scans. That approach is fully in concordance with the FIGO recommendation (2). Published studies report the lower incidence of late toxicities after radical combined RT, usually about $10-12 \%$ of Grade III-IV side effects $(6,7)$. It is necessary to enhance a fact that in our study, in $12 \%$ of patients who underwent radical RT serious late complications developed more than 5 years since RT, thus the first 5 years after RT they had lived without complications and side-effects. Studies published data of 10 years or even longer follow-up are exceptional. In the study of Misra et al. (8), the authors compared late toxicities related to radical RT or radical chemoradiotherapy during the mean 10 years of clinical follow-up. They found late toxicities grade III-IV in about $21 \%$ and $25 \%$, respectively. They reported about $7.5 \%$ of rectal, $15 \%$ of bladder, $3 \%$ of small bowel and $5 \%$ of bone toxicities. They also reported $3.8 \%$ morbidity related to radiation related side effects (8). Our data were understandably higher, because we studied patients reported some clinical complaints and underwent imaging scans, we did not evaluate complete cohorts of treated females.

In our study we found one case of an uretero-arterial fistula, which is a very rare life-threatening complication. This case of serious very late radiation-related toxicity appeared 10 years since RT. There are about 150 cases of uretero-arterial fistulas reported in the literature (9). Most of cases (about $85 \%$ ) are secondary, related to previous surgery, RT or trauma (9). The mortality rate of ureteroarterial fistulas is reported between $7-23 \%$ and an urgent intervention is always needed (9). We successfully treated our patient by a covered stent placement; more details are described in the Case report 2.

Radiation-induced tumors must be considered as very late side effects (10). We found one case of undifferentiated bone sarcoma after radical combined RT, which developed in the field of osteonecrosis and was diagnosed 22 years after RT. Detailed history of that case is provided as the Case report 1. The case fulfills the Cahan's criteria for radiation induced tumors (11). The Cahan's criteria are as follow: a tumor must be situated in a field of RT, with sufficient latent period and previously treated cervical carcinoma must be of a different histological origin, affected organs must be morphologically normal before RT (11). Carcinomas as secondary radiationinduced tumors are commonly seen in organs receiving low dose radiation; whereas sarcomas are predominantly seen arising in tissues/organs receiving high dose of radiation (12). According to Hall and Wuu (12) a relative risk of development of radiation-induced malignancies after RT for cervical cancer is as follows: bladder carcinoma 4.5 , vaginal cancer 2.7 and bone tumors 1.3.

Bone toxicities belong to the next late/very late radiation-induced side effects. It is a well-known fact that the bone marrow is a very sensitive tissue and bone marrow fat replacement is common pathophysiological reaction, which is apparent in the site of $\mathrm{RT}$ in all irradiated patients $(13,14)$. Bone comorbidities are not an infrequent consequence of gynecological cancer treatment as a result of decreased bone mineralization, elastic resistance and even radiation-induced osteonecrosis (15). Some studies proposed that concurrent chemotherapy increases a rate of the bone toxicities $(8,16)$. In the study of Misra et al. $(8)$, patients treated by chemoradiotherapy suffered from bone toxicities grade III-IV in $25 \%$, while patients underwent only radical RT in $16 \%$. We found osteonecrosis or insufficiency fractures in $9(22 \%)$ females who underwent radical $\mathrm{RT} \pm$ chemotherapy, 4 cases of them were clinically silent. Schmeler et al. diagnosed pelvic fractures after RT in $9.7 \%$ of patients; $38 \%$ of patients were diagnosed within the first year after RT and $83 \%$ were diagnosed within the second year after completing therapy (16). In their study only $45 \%$ of fractures were symptomatic (16). According to the retrospective MRI study by Kwon et al. the 5-year cumulative prevalence of insufficiency fractures after RT for cervical cancer was $45.2 \%$, the median time of an insufficiency fracture diagnosis was 16.9 months after RT (13). Insufficiency fractures or osteonecrosis in our patients were diagnosed in remarkably longer period, the median time since RT was about 16 years. However, we must clearly state, that our females did not undergo regular imaging scans after RT, so in most of them we do not know what time bone complications exactly appeared. If we count all diagnosed late bone toxicities bone sarcoma included, nearly $45 \%$ of them was asymptomatic. Our results are similar to data reported by Schmeler et al., $55 \%$ of late bone complications were asymptomatic in their study (16). Considering our results and the literature data we came to conclusion that the late bone toxicities are probably underdiagnosed. 
Our study has several important limitations. The first limitation is its retrospective design. However, we consider prospective design with so long follow-up (median 14 years) as nearly impossible. Secondly, the study group was highly selected; we choose subjects who reported some complaints and therefore were sent to cross-sectional imaging scans. That means our data must not be taken as an incidence evaluation, but as an observation of side-effects in symptomatic patients. The study cohort was also limited.

\section{Conclusions}

Our study shows that if young females successfully treated for advanced cervical cancer by RT report abdominal/ pelvic problems, it is highly probable their imaging scans will reveal late radiation related side effects. These comorbidities may often affect the rest of their life. Our study puts forward question whether females after radical RT should have been regularly followed by cross-sectional methods.

\section{Acknowledgments}

Funding: This study was support by Charles University research program PROGRES Q28.

\section{Footnote}

Conflicts of Interest: All authors have completed the ICMJE uniform disclosure form (available at http://dx.doi. org/10.21037/qims-20-553). HM serves as an unpaid editorial board member of Quantitative Imaging in Medicine and Surgery. The other authors have no conflicts of interest to declare.

Ethical Statement: All patients of our institution provided written, informed consent to treatment included diagnostic procedures (as CT, PET/CT and MRI) and agreed with publishing medical data all images included in scientific literature in anonymous form. The informed consent forms are deposit in their personal files. This retrospective study conforms to the Declaration of Helsinki and was approved by the Ethics committee of Faculty Hospital Kralovske Vinohrady (EK-VP/22/00/2019), Prague, Czech Republic.

Open Access Statement: This is an Open Access article distributed in accordance with the Creative Commons Attribution-NonCommercial-NoDerivs 4.0 International
License (CC BY-NC-ND 4.0), which permits the noncommercial replication and distribution of the article with the strict proviso that no changes or edits are made and the original work is properly cited (including links to both the formal publication through the relevant DOI and the license). See: https://creativecommons.org/licenses/by-nc-nd/4.0/.

\section{References}

1. Ferlay J, Soerjomataram I, Dikshit R, Eser S, Mathers C, Rebelo M, Parkin DM, Forman D, Bray F. Cancer incidence and mortality worldwide: sources, methods and major patterns in GLOBOCAN 2012. Int J Cancer 2015;136:E359-86.

2. Bhatla N, Aoki D, Sharma DN, Sankaranarayanan R. Cancer of the cervix uteri. Int J Gynaecol Obstet 2018;143 Suppl 2:22-36.

3. Viswanathan AN, Lee LJ, Eswara JR, Horowitz NS, Konstantinopoulos PA, Mirabeau-Beale KL, Rose BS, von Keudell AG, Wo JY. Complications of pelvic radiation in patients treated for gynecologic malignancies. Cancer 2014;120:3870-83.

4. Singh GK, Yadav V, Singh P, Bhowmik KT. RadiationInduced Malignancies Making Radiotherapy a "TwoEdged Sword": A Review of Literature. World J Oncol 2017;8:1-6.

5. Common terminology criteria for adverse events v5.0. Available online: https://www.meddra.org/

6. Castelnau-Marchand P, Chargari C, Maroun P, Dumas I, Del Campo ER, Cao K, Petit C, Martinetti F, TafoGuemnie A, Lefkopoulos D, Morice P, Haie-Meder C, Mazeron R. Clinical outcomes of definitive chemoradiation followed by intracavitary pulsed-dose rate image-guided adaptive brachytherapy in locally advanced cervical cancer. Gynecol Oncol 2015;139:288-94.

7. Ribeiro I, Janssen H, De Brabandere M, Nulens A, De Bal D, Vergote I, Van Limbergen E. Long term experience with 3D image guided brachytherapy and clinical outcome in cervical cancer patients. Radiother Oncol 2016;120:447-54.

8. Misra S, Lal P, Kumar Ep S, Rastogi N, Tiwari A, Singh S, Das KJM, Kumar S. Comparative assessment of late toxicity in patients of carcinoma cervix treated by radiotherapy versus chemo-radiotherapy - Minimum 5 years follow up. Cancer Treat Res Commun 2018;14:30-6.

9. Pillai AK, Anderson ME, Reddick MA, Sutphin PD, Kalva SP. Ureteroarterial fistula: diagnosis and management. AJR Am J Roentgenol 2015;204:W592-8. 
10. Braunstein S, Nakamura JL. Radiotherapy-induced malignancies: review of clinical features, pathobiology, and evolving approaches for mitigating risk. Front Oncol 2013;3:73.

11. Cahan WG, Woodard HQ, Higinbotham NL, Stewart FW, Coley BL. Sarcoma arising in irradiated bone: report of eleven cases. Cancer 1998;82:8-34.

12. Hall EJ, Wuu CS. Radiation-induced second cancers: the impact of 3D-CRT and IMRT. Int J Radiat Oncol Biol Phys 2003;56:83-8.

13. Kwon JW, Huh SJ, Yoon YC, Choi SH, Jung JY, Oh $\mathrm{D}$, Choe BK. Pelvic bone complications after radiation therapy of uterine cervical cancer: evaluation with MRI.

Cite this article as: Malikova $\mathrm{H}$, Burghardtova $\mathrm{M}$, Fejfarova $\mathrm{K}$, Nadova K, Weichet J. Advanced cervical cancer in young women: imaging study of late and very late radiation-related side effects after successful treatment by combined radiotherapy. Quant Imaging Med Surg 2021;11(1):21-31. doi: 10.21037/qims-20-553
AJR Am J Roentgenol 2008;191:987-94.

14. Papadopoulou I, Stewart V, Barwick TD, Park WH, Soneji N, Rockall AG, Bharwani N. Post-Radiation Therapy Imaging Appearances in Cervical Carcinoma. Radiographics 2016;36:538-53.

15. Lundin B, Björkholm E, Lundell M, Jacobsson $H$. Insufficiency fractures of the sacrum after radiotherapy for gynaecological malignancy. Acta Oncol 1990;29:211-5.

16. Schmeler KM, Jhingran A, Iyer RB, Sun CC, Eifel PJ, Soliman PT, Ramirez PT, Frumovitz M, Bodurka DC, Sood AK. Pelvic fractures after radiotherapy for cervical cancer: implications for survivors. Cancer 2010;116:625-30. 